\title{
Aplicación de la técnica del vecino más cercano para la solución del problema del agente viajero y minimizar los costos de operación
}

\section{Application of the closest neighbor technique for the solution of the traveler agent problem and minimize operating costs}

\author{
Marcelino García-Miguel ${ }^{a}$, Arturo Contreras-Juárez ${ }^{b}$, Ana Karen González-Vásquez ${ }^{c}$, \\ Wendolin Hernández-López ${ }^{d}$. Alan Cova-Cervantes ${ }^{e}$
}

\begin{abstract}
:
The companies of road vehicle transport, face a challenge every day to control the operating costs, with the emergence of the sanitary pandemic has been more affected by the decrease of the movement of cargo and the increase of the cost of fuel, so this article sets out how to design pre-routes for this mode of transport, solving the problem of the travel agent through the technique of the nearest neighbour, with the support of google maps, to minimize the operating costs in any company that has the need to make routes with road vehicle transport and does not have a digital platform for the design of its routes for the investment costs that this represents.

Keywords:

Travel Agent Problem, Google Maps, Operating Costs, Nearest Neighbor Technician

\section{Resumen:}

Las empresas de transporte vehicular terrestre, enfrentan un desafío cada día por controlar los costos de operación, con el surgimiento de la pandemia sanitaria se ha visto más afectado por la disminución de movimiento de carga y el incremento del costo de combustible, por lo que el presente artículo expone la forma de diseñar pre rutas para este medio de transporte, dando solución al problema del agente viajero a través de la técnica del vecino más cercano, con apoyo de google maps, para minimizar los costos de operación en cualquier empresa que tenga la necesidad de hacer recorridos con el transporte vehicular terrestre y no cuente con una plataforma digital para el diseño de sus recorridos por los costos de inversión que este representa.
\end{abstract}

\section{Palabras Clave:}

Problema del agente viajero, Google Maps, Costos de operación, Técnica vecino más cercano

\section{Introducción}

El transporte terrestre vehicular, es el modo de transporte que mueve la mayor cantidad de carga, en México representa más del $50 \%$ del movimiento total nacionaly genera el $49 \%$ de los empleos en el sector transporte. Por otra parte, hay más de 400,000 vehículos destinados para el trayecto por carretera, de esta manera se ratifica el desarrollo competitivo del transporte de carga en la logística, dentro de la cadena de suministro estableciendo su crecimiento y desarrollo a fin de generar estrategias que generen apoyo a una logística integral en el transporte de carga. Mientras que el transporte de carga terrestre es de suma importancia para el sector productivo nacional. Al mismo tiempo el transporte es la actividad fundamental para la generación de empleo y

aUniversidad Politécnica de Tlaxcala Región Poniente, https://orcid.org/0000-0001-6139-4443, Email: $\underline{\text { marcelinogarcia@uptlaxponiente.edu.mx }}$

bAutor de Correspondencia. Universidad Politécnica de Tlaxcala Región Poniente, https://orcid.org/0000-0002-7192-3596, Email: arturocontreras@uptlaxponiente.edu.mx

c Universidad Politécnica de Tlaxcala Región Poniente, https://orcid.org/0000-0003-3727-1237, Email: agonzalezvasq07@gmail.com dUniversidad Politécnica de Tlaxcala Región Poniente, https://orcid.org/0000-0002-6020-5089, Email: wendo0521@gmail.com 
equilibrio social, así como un factor importante de productividad, competitividad y crecimiento económico nacional, y determina el acarreo de carga por carretera como principal modo de transporte (Contreras, 2020).

Por otra parte, el transporte de carga contribuye a una congestión, conectando en muchos niveles a personas y bienes, de lo local a lo global aumentando las emisiones y el ruido en las calles. En Estados Unidos de América, los camiones generan el $18 \%$ del costo de la congestión, aunque sólo representan el $7 \%$ de los viajes urbanos (Rodriguez, 2016).

De acuerdo al Instituto Mexicano del Transporte "La dispersión del COVID-19 a través de los corredores de transporte carretero en México", para el transporte terrestre los camiones de carga representan el $7.9 \%$ del total de los vehículos motorizados que cruzan la frontera norte, con una cifra mensual que fluctúa alrededor de las 550 mil unidades, donde casi el $72 \%$ viajan cargados y el $28 \%$ restante circulan vacíos. Mientras que para los puertos y fronteras fue distinto ya que, durante el primer trimestre del presente año, alcanzó una tasa de crecimiento de $6.2 \%$ (Transporte, 2020).

Dado lo anterior, se estima que, de 1, 873,564 empresas en el país, poco más de la mitad de éstas instrumentaron paros técnicos como medida de prevención, mientras que $40.4 \%(757,581)$ no lo hicieron. Las microempresas fueron quienes en su mayoría llevaron a cabo esta acción registrando $93.4 \%$. Los periodos de tiempo muestran en gran medida el movimiento y ampliación de la contingencia (Inegi, 2020).

Con el seguimiento a los datos se estima que, se han perdido 346 mil 878 empleos, esto, en el periodo de la contingencia por el coronavirus, del 13 de marzo al 6 de abril, los estados donde hubo más pérdida de empleo fue Quintana Roo, con 63 mil 847, Ciudad de México, con 55 mil 591, Nuevo León, con 23 mil 465, Jalisco, con 21 mil 535, Estado de México, con 16 mil 36 y Tamaulipas, con 12 mil 652. Son 31 empresas relacionadas con el comercio, construcción, transportes, comunicaciones y de servicios las cuales registraron la pérdida total de empleo (Universal, 2020).

Cada día, estima, la Confederación de Cámaras Industriales (CONCAMIN) se pierden 20 mil trabajos (Cota, 2020).

De acuerdo a los datos recuperados y presentados el brote del COVID-19 vino a originar un desequilibrio total en las empresas y generó un desequilibrio importante en el diseño y seguimiento de las rutas del transporte terrestre por desistir de software de altos costos. Por lo que es importante conjugar software de acceso libre y técnicas de recorrido para el transporte a fin de ayudar a estas empresas a minimizar sus costos de operación. Google maps es un software de acceso gratuito y fue diseñado por Lars y Jeans Eilstrup Rasmussen, este es un servicio que ofrece Google mediante el cual se puede visualizar mapas e imágenes satelitales desde cualquier parte del mundo, contiene una variedad de herramientas de búsqueda y configuración para que el usuario pueda interactuar con el lugar deseado, por otra parte, puede realizar el cálculo de la distancia de dos ubicaciones diferentes (Otamendi, 2014).

Google maps se considera como una fuente de información por localización geográfica de su propio buscador web, que están dadas por el sistema del WGS84, que es la información que brindan los satélites estadounidenses por sus siglas en inglés World Geodetic System 84 (Sistema Geodésico Mundial 1984). Esté utiliza la información para mostrar la latitud y longitud en todos los puntos cardenales con un error de cálculo menor a $2 \mathrm{~cm}$., y por este último punto es la razón por la cual se basa el Sistema de Posicionamiento Global (Chillagano, 2017).

A está aplicación de mapas se le pueden dar diferentes usos, uno de los principales es el de consultar direcciones y calcular rutas; se puede averiguar cuál es la ubicación de cualquier lugar, así como calcular la ruta desde diferentes puntos. Puede indicar la dirección a tomar de camino a un lugar, identificando y dando seguimiento a su desplazamiento. Otra de sus funciones es la de consultar en detalle cómo es un territorio gracias a las fotos por satélite, así como la comprobación orográfica del terreno gracias a la opción disponible para ver el relieve de un mapa (Ravea, 2012).

Por lo tanto, Google Maps proporciona una interfaz de programa de aplicación, para representar un servicio de mapas en una aplicación personalizada. Este se ha convertido en unos de los productos más importantes. Estos beneficios que nos brinda se justifican no solo por las características y el rendimiento, sino también por su capacidad para integrar diferentes lenguajes de programación, con el mando de mejorar la personalización y la explotación de diferentes sistemas. Desde las perspectivas de las herramientas de google maps se han desarrollado prototipos que permiten generar georreferenciación al usuario, especificando ubicaciones con exactitud y acceder de forma rápida, con el objetivo de optimizar los recursos y reducir los costos en el proceso de recopilación de información y así conocer tu vecino más cercano (Battin, 2016). 
Con esta herramienta tecnológica de apoyo se pretende dar solución al problema del agente viajero o TSP por sus siglas en inglés (Travelling Salesman Problem), el cual es uno de los problemas más famosos y complejos, de las ciencias computacionales y han sido abordados por varias ramas de la Ingeniería y por distintas razones, su principal aplicación es la de generar rutas desde distintas perspectivas, ya sea un proceso que lleva una secuencia específica o una distribución de carácter logístico en la que intervienen elementos de transporte buscando la mejor ruta posible, con la aplicación de la técnica del vecino más cercano, la cual es una técnica que genera rutas uniendo vértices o puntos, teniendo en cuenta la menor arista o distancia euclidiana de un punto a los demás puntos, esta secuencia de inserción de puntos, inicia desde el depósito, incorporando el punto más cercano, después de ser incluido se inicia la búsqueda del siguiente punto a ser incluido, con la menor distancia al anterior punto incluido, para la incorporación de los puntos se puede tener en cuenta la restricción de capacidad del vehículo, de distancia total de ruta, de tiempo total de ruta o la combinación de estas tres (Páez, 2016).

\section{Materiales y métodos}

Dentro de los problemas más conocidos, en el ámbito de la planeación de rutas se encuentra el problema del agente viajero, y el problema de la planeación de rutas vehiculares para los cuales se han desarrollado diversos algoritmos que buscan la solución óptima con el menor costo computacional. Por tal motivo, se proponen alternativas para el mejor uso de los recursos. Estas disciplinas que abordan este tema son la investigación de operaciones y las ciencias informáticas como algoritmia y teoría de grafos (López, 2013).

El problema del agente viajero se define dado un entero $n>\emptyset$ y las distancias entre cada par de las $n$ ciudades, estas distancias se da por medio de la matriz ( $d i j)$ de dimensión $n \times n$, donde ( $d i j$ ) es un entero mayor o igual a cero. Un recorrido es una trayectoria que visita todas las ciudades exactamente una vez. El problema consiste en encontrar un recorrido con longitud total mínima. Una permutación $\pi$ cíclica representa un recorrido, si se interpreta $\pi$ (j) como la ciudad después de la ciudad $j=$ $1,2, \ldots n)$. (Cobos, 2014).

EI TSP formula un agente viajero, partiendo de su ciudad de origen, debe visitar exactamente una vez cada ciudad de un conjunto de ellas (previamente especificado), y retornar al punto de partida. Un recorrido con estas características, es llamado dentro de este contexto un tour. Para simplificar podríamos decir que el problema consiste en encontrar el tour para el cual la distancia total recorrida sea mínima, se asume que se conoce, para cada par de ciudades, la distancia entre ellas. Donde cada nodo del grafo corresponde a una ciudad y cada arista que une a un par de nodos representa la parte del tour que pasa por dichos nodos coordenadas de las ciudades (Villegas, 2017).

El problema en si es fácil de formular, sin embargo, pertenece a una clase de problemas muy difíciles de resolver, en el entendido que resolver significa hallar la solicitud óptima (Mendoza, 2017).

El algoritmo del agente viajero se representa con una distancia de recorrido entre ciudades que puede ser representada en metros, kilómetros, millas, milímetros, así como también un costo de traslado. Las variables que se pueden aportar dependen de cada problema de las cuales se presentan casos de éxito en la aplicación del algoritmo del agente viajero como: Aplicación del problema del agente viajero a los principales hoteles de la ciudad de Barranquilla, (Arias, 2017).

Algoritmo del agente viajero para minimizar el recorrido de visitas programadas en una empresa de seguros, (Reyes, 2017).

Solución al Problema de Secuenciación de Trabajos mediante el Problema del Agente Viajero, (Fuentes, 2016).

La aplicación de la técnica del vecino más cercano, busca hacer una serie de visitas a diferentes clientes 0 nodos sin pasar por el mismo nodo o cliente dos veces a fin de minimizar el tiempo o kilometraje de recorrido; trata de un procedimiento constructivo, se parte de elegir un vértice inicial, Ilamado $j 1$. Una vez seleccionado, se mide la distancia que hay de este vértice a los restantes, y se elige aquél cuya distancia al vértice inicial sea la mínima (es decir al vecino más cercano), y se llama j2. De la misma forma, se construye una trayectoria $j 1, j 2, j 3, \ldots j k, j k+1, \ldots j n$ donde el vértice $j k+1$ se elige tomando la mínima distancia que hay desde $j k$ hasta cada uno de los vértices que sean distintos de los ya elegidos $j 1, j 2, j 3, j k$. Al terminar, se debe de agregar el arco que va del vértice $j n$, hasta el vértice $j 1$. Con esto se ha completado el tour (Arriagada, 2015).

\section{Diseño de rutas para una empresa de venta de material hemerográfico}

Dadas las herramientas y métodos se diseña un recorrido para una empresa dedicada a la venta de material hemerográfico a institu ciones edu cativas a nivel nacional 
que se encuentra ubicada en Calle 5 poniente No. 190119. Col. Centro. Puebla, Pue. La cual reporta no tener un control sobre los costos de operación de las rutas que recorre su personal de ventas dejando que los mismos agentes reporten sus gastos y estos son cubiertos por la empresa. La Tabla 1 Presenta los recorridos de las visitas generadas por el agente viajero en un periodo de tiempo.

Tabla 1.1 Visita de clientes presentada por la empresa.

\begin{tabular}{|c|c|c|}
\hline $\begin{array}{l}\text { No. } \\
\text { De } \\
\text { visitas }\end{array}$ & Clientes & Dirección \\
\hline 1 & $\begin{array}{l}\text { Universidad } \\
\text { Politécnica de } \\
\text { Tulancingo }\end{array}$ & $\begin{array}{l}\text { Calle Ingenierías No. } 100 . \text { Col. } \\
\text { Huapalcalco. CP. } 43629 . \\
\text { Tulancingo de Bravo, Hidalgo. }\end{array}$ \\
\hline 2 & $\begin{array}{l}\text { Centro Universitario } \\
\text { Metropolitano }\end{array}$ & $\begin{array}{l}\text { Blvd. Nuevo Hidalgo 509-A, } \\
\text { Plaza Gran Sur. Col. Las } \\
\text { Colonias Tulipanes II. CP. } \\
\text { 42083, Pachuca de Soto, } \\
\text { Hidalgo. }\end{array}$ \\
\hline 3 & $\begin{array}{l}\text { Tecnológico de } \\
\text { Monterrey Campus } \\
\text { Hidalgo }\end{array}$ & $\begin{array}{l}\text { Blvd. Felipe Angeles 2003. Col. } \\
\text { Venta Prieta. CP. } 42080 . \\
\text { Pachuca de Soto, Hidalgo. }\end{array}$ \\
\hline 4 & $\begin{array}{l}\text { Universidad } \\
\text { Tecnológica de } \\
\text { Tulancingo }\end{array}$ & $\begin{array}{l}\text { Camino a Ahuehuetitla, } 301 . \\
\text { Col. Las Presas Ahuehuetitla. } \\
\text { CP. 43645, Tulancingo de } \\
\text { Bravo, Hidalgo. }\end{array}$ \\
\hline 5 & $\begin{array}{l}\text { Centro Universitario } \\
\text { Siglo XXI }\end{array}$ & $\begin{array}{l}\text { Blvd. Felipe Ángeles } 403 . \\
\text { Ampliación Santa Julia. CP. } \\
42080, \text { Pachuca de Soto, } \\
\text { Hidalgo. }\end{array}$ \\
\hline 6 & Colegio Anáhuac & $\begin{array}{l}\text { Nicolo Paganini 303. Col. } \\
\text { Jardines del Sur. CP. } 43660 . \\
\text { Tulancingo de Bravo, Hidalgo. }\end{array}$ \\
\hline 7 & $\begin{array}{l}\text { Universidad la Salle } \\
\text { de Pachuca }\end{array}$ & $\begin{array}{l}\text { Belisario Domínguez } 202 \\
\text { Centro. CP. 43830, Pachuca de } \\
\text { Soto, Hidalgo. }\end{array}$ \\
\hline 8 & $\begin{array}{l}\text { Centro Universitario } \\
\text { del Oriente de } \\
\text { Hidalgo }\end{array}$ & $\begin{array}{l}\text { Av. Juárez Sur 101-B Centro. } \\
\text { CP. } 43600 \text {, Tulancingo de } \\
\text { Bravo, Hidalgo. }\end{array}$ \\
\hline 9 & $\begin{array}{l}\text { Universidad } \\
\text { Politécnica de } \\
\text { Pachuca }\end{array}$ & $\begin{array}{l}\text { Carretera Pachuca-Cd. } \\
\text { Sahagún Km 20. Ex Hacienda } \\
\text { de Santa Bárbara. CP. } 43830 . \\
\text { Pachuca de Soto Hidalgo. }\end{array}$ \\
\hline 10 & $\begin{array}{l}\text { Universidad ETAC } \\
\text { Campus Tulancingo }\end{array}$ & $\begin{array}{l}\text { Av. } 21 \text { de marzo } 511 \text { esq. } 16 \text { de } \\
\text { septiembre Centro. CP. } 43600 \text {, } \\
\text { Tulancingo de Bravo, Hidalgo. }\end{array}$ \\
\hline
\end{tabular}

La Tabla 2 presenta las variables de control que son los indicadores que generan el costo del viaje, el tiempo y kilometraje recorrido que fueron reportados por el agente resultado de visitar a todos los clientes.

Tabla 2 Información de las vistas del agente viajero.

\begin{tabular}{lc}
\hline \multicolumn{1}{c}{ Variables de control } & $\begin{array}{c}\text { Reporte de Agente } \\
\text { Viajero }\end{array}$ \\
\hline \hline Km recorrido total & 1,014 \\
Tiempo total empleado (minutos) & 1,440 \\
Costo de combustible & $\$ 1,560.00$ \\
Viáticos para agente & $\$ 900.00$ \\
\hline \hline
\end{tabular}

Fuente: Elaboración propia de datos proporcionados por la empresa.

De acuerdo a las rutas generadas por el agente y los datos de las variables de control presentadas se pretende generar las mismas visitas bajo el esquema de la técnica del vecino más cercano con apoyo de google Maps para la toma de distancias y tiempos.

De acuerdo a los datos proporcionados sobre los materiales y métodos utilizados en la solución del problema se formula su aplicación en 4 puntos que se describen a continuación:

1.- Se planea el recorrido un día antes aprovechando visitas a clientes, nuevos prospectos y entrega de productos, generando una tabla de datos con las direcciones llamadas nodos como lo menciona la técnica del vecino más cercano.

2.- Se toman las distancias de cada una de las direcciones (nodos), y se genera una matriz de distancia entre todas las direcciones conocidas como aristas.

3.- $S e$ reconoce el punto de partida que en este caso es el punto de inicio de las instalaciones de la empresa.

4.- De acuerdo al método (Técnica del vecino más cercano) se busca la distancia más corta del punto de inicio al primer cliente (nodo 1), posteriormente este nodo 1 se convierte en el punto de inicio y se hace nuevamente una revaloración de las distancias, de esta forma se traslada al cliente 2 (nodo 2), y así sucesivamente hasta hacer la última visita, y regresar al punto de inicio mejor conocido como las instalaciones de la empresa.

\section{Resultados}

Dando seguimiento al planteamiento de la solución y considerando el punto número 1 del método formulado, se dividieron en dos secciones las visitas una para los clientes del municipio de Tulancingo y otra para los de Pachuca como lo muestra la Tabla 3.

Tabla 3 División de visitas por municipio.

\begin{tabular}{|cl|}
\hline No. De visitas & \multicolumn{1}{c}{ Clientes } \\
\hline 1 & TULANCINGO HIDALGO \\
2 & Universidad Politécnica de Tulancingo \\
3 & Centro Universitario del Oriente de Hidalgo \\
4 & Universidad ETAC Campus Tulancingo \\
5 & Universidad Tecnológica de Tulancingo \\
\hline 1 & PACHUCA HIDALGO \\
2 & Centro Universitario Metropolitano \\
\end{tabular}




\begin{tabular}{|c|c|}
\hline 3 & Tecnológico de Monterrey Campus Hidalgo \\
\hline 4 & Centro Universitario Siglo XXI \\
\hline 5 & Universidad la Salle de Pachuca \\
\hline
\end{tabular}

Se da continuidad con la aplicación de la tecnología de información Google maps y atendiendo al punto número 2 del método de solución. La Figura 1 presenta una muestra de la obtención de las distancias entre la empresa y cada uno de los clientes, y la Tabla 4 presenta la matriz de distancias entre la empresa y cada uno de los clientes.

Figura 1. Calculo de las distancias de los clientes.

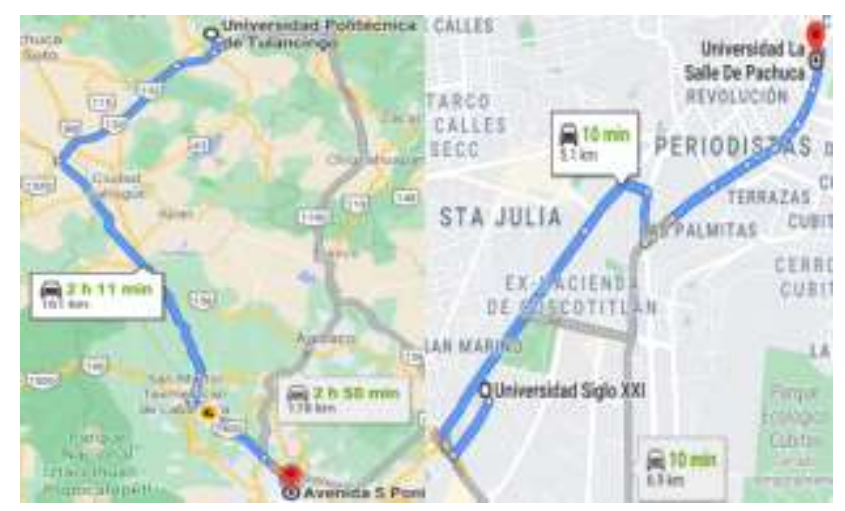

Fuente: Datos obtenidos de Google Maps.

Tabla 4 Matriz de distancias ( $\mathrm{km}$ ) entre clientes y la

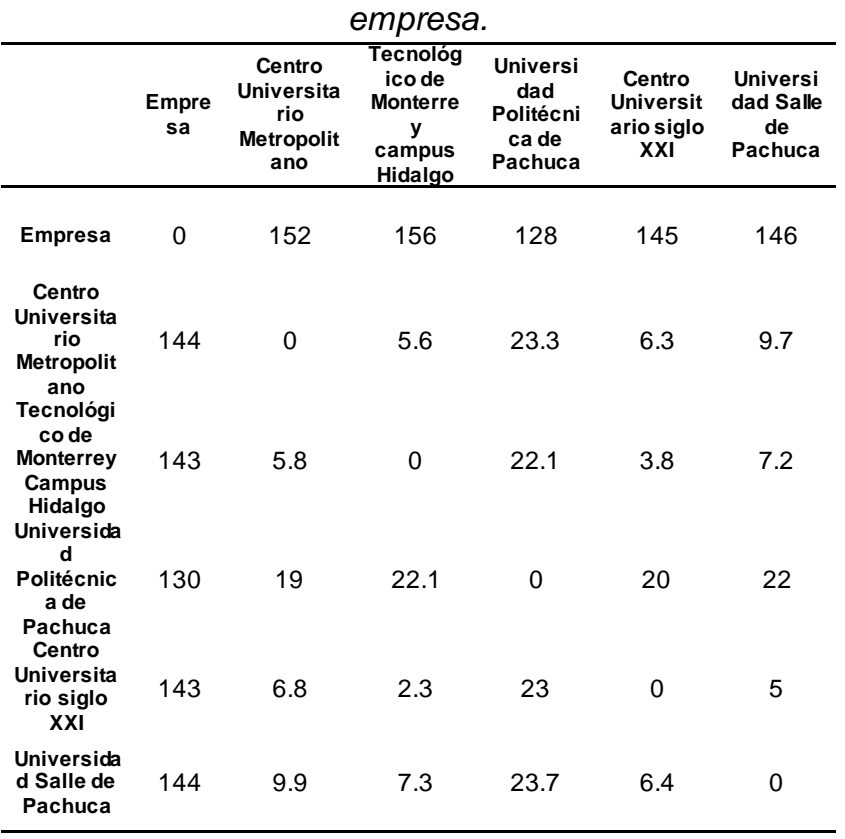

\begin{tabular}{|c|c|c|c|c|c|}
\hline $\begin{array}{c}\text { Empre } \\
\text { sa }\end{array}$ & $\begin{array}{c}\text { Centro } \\
\text { Universita } \\
\text { rio del } \\
\text { Oriente } \\
\text { Hidalgo }\end{array}$ & $\begin{array}{l}\text { Colegio } \\
\text { Anáhuac }\end{array}$ & $\begin{array}{c}\text { Universi } \\
\text { dad } \\
\text { Tecnológ } \\
\text { ica de } \\
\text { Tulancin } \\
\text { go }\end{array}$ & $\begin{array}{c}\text { Universid } \\
\text { ad } \\
\text { Politécnic } \\
\text { a de } \\
\text { Tulancing } \\
\text { o }\end{array}$ & $\begin{array}{c}\text { Universi } \\
\text { dad } \\
\text { ETAC } \\
\text { Campus } \\
\text { Tulancin } \\
\text { go }\end{array}$ \\
\hline
\end{tabular}

\begin{tabular}{|c|c|c|c|c|c|c|}
\hline Empresa & 0 & 156 & 155 & 154 & 162 & 157 \\
\hline $\begin{array}{c}\text { Centro } \\
\text { Universita } \\
\text { rio del } \\
\text { Oriente } \\
\text { Hidalgo }\end{array}$ & 155 & 0 & 1 & 4.6 & 7.6 & 1.5 \\
\hline $\begin{array}{l}\text { Colegio } \\
\text { Anáhuac }\end{array}$ & 154 & 1.2 & 0 & 3.9 & 7.9 & 2.1 \\
\hline $\begin{array}{c}\text { Universida } \\
d \\
\text { Tecnológi } \\
\text { ca de } \\
\text { Tulancing } \\
\text { o }\end{array}$ & 151 & 5.3 & 3.8 & 0 & 11.4 & 6 \\
\hline $\begin{array}{c}\text { Universida } \\
d \\
\text { Politécnic } \\
\text { a de } \\
\text { Tulancing } \\
\text { o }\end{array}$ & 161 & 7.4 & 8.2 & 11.1 & 0 & 7.3 \\
\hline $\begin{array}{c}\text { Universida } \\
\text { d ETAC } \\
\text { Campus } \\
\text { Tulancing } \\
\text { o }\end{array}$ & 156 & 1 & 2.2 & 5.8 & 7.2 & 0 \\
\hline
\end{tabular}

De acuerdo a la matriz de distancias generadas se aplica la técnica del vecino más cercano del cual se marcan las distancias más cortas tomando como nodo de partida la empresa generando el recorrido y regresando al nodo o punto de partida, donde la figura 2 presenta la ruta que ha de seguir el agente viajero, misma que se da seguimiento a través de la aplicación de Google Maps lo que permite que el agente no genere alguna desviación de la ruta y así maximiza las operaciones de visita a los clientes de la empresa. La Tabla 5 ratifica el recorrido el cual es entregado impreso al agente para su seguimiento y cumplimiento.

Tabla 5 Tabla de recorrido y de entrega al agente viajero.

\begin{tabular}{|c|c|c|c|c|}
\hline No. & ORIGEN & DESTINO & KM & $\begin{array}{c}\text { TIEMPO } \\
\text { (hrs.) }\end{array}$ \\
\hline 1 & Empresa & $\begin{array}{l}\text { Universidad Politécnica de } \\
\text { Pachuca }\end{array}$ & 128 & $1: 45$ \\
\hline 2 & $\begin{array}{l}\text { Universidad Politécnica de } \\
\text { Pachuca }\end{array}$ & $\begin{array}{l}\text { Centro Universitario } \\
\text { Metropolitano }\end{array}$ & 19 & $0: 22$ \\
\hline 3 & $\begin{array}{l}\text { Centro Universitario } \\
\text { Metropolitano }\end{array}$ & $\begin{array}{l}\text { Tecnológico de Monterrey } \\
\text { Campus Hidalgo }\end{array}$ & 5.6 & $0: 12$ \\
\hline 4 & $\begin{array}{l}\text { Tecnológico de Monterrey } \\
\text { Campus Hidalgo }\end{array}$ & $\begin{array}{l}\text { Centro Universitario siglo } \\
\text { XXI }\end{array}$ & 3.8 & 0:09 \\
\hline 5 & $\begin{array}{l}\text { Centro Universitario siglo } \\
\text { XXI }\end{array}$ & $\begin{array}{l}\text { Universidad Salle de } \\
\text { Pachuca }\end{array}$ & 5 & $0: 10$ \\
\hline 6 & $\begin{array}{l}\text { Universidad Salle de } \\
\text { Pachuca }\end{array}$ & Empresa & 144 & $1: 56$ \\
\hline \multicolumn{3}{|c|}{ TOTAL } & 305.4 & $4: 34$ \\
\hline No. & ORIGEN & DESTINO & KM & $\begin{array}{c}\text { TIEMPO } \\
\text { (hrs.) }\end{array}$ \\
\hline 1 & Empresa & $\begin{array}{l}\text { Universidad Tecnológica de } \\
\text { Tulancingo }\end{array}$ & 154 & $2: 04$ \\
\hline 2 & $\begin{array}{l}\text { Universidad Tecnológica de } \\
\text { Tulancingo }\end{array}$ & Colegio Anáhuac & 3.8 & $0: 08$ \\
\hline
\end{tabular}




\begin{tabular}{lllcc} 
& Colegio Anáhuac & $\begin{array}{l}\text { Centro Universitario del } \\
\text { Oriente Hidalgo }\end{array}$ & 1.2 & $0: 06$ \\
4 & $\begin{array}{l}\text { Centro Universitario del } \\
\text { Oriente Hidalgo } \\
\text { Universidad ETAC Campus } \\
\text { Tulancingo }\end{array}$ & 1 & $0: 05$ \\
& $\begin{array}{l}\text { Universidad ETAC Campus } \\
\text { Tulancingo } \\
\text { Universidad Politécnica de }\end{array}$ & 7.2 & $0: 16$ \\
& $\begin{array}{l}\text { Universidad Politécnica de } \\
\text { Tulancingo }\end{array}$ & Empresa & 161 & 2,11 \\
\hline & TotaL & $\mathbf{3 2 8 . 2}$ & $\mathbf{4 : 5 0}$ \\
\hline
\end{tabular}

Fuente. Elaboración Propia de datos obtenidos de la Figura 2.

Para los recorridos de visita que se consideraron y afín de validar los resultados de la aplicación de los métodos, se utiliza un mismo vehículo de la marca Nissan en su modelo Tsuru año 2017 de 4 cilindros de 1.6 litros, con un rendimiento de combustible y desplazamiento promedio de $15 \mathrm{~km} / \mathrm{l}$.

La Figura 2 presenta el diagrama de recorrido de los clientes de Pachuca y posteriormente el de Tulancingo, se visualiza en primera instancia la figura con los nodos en sentido ordenado de visita y el kilómetro entre nodos o clientes, por otra parte, se presenta la imagen obtenida de google maps del recorrido que se realizó por el agente.

Figura 2. Ruta que se genera con la aplicación de la técnica del vecino más cercano.
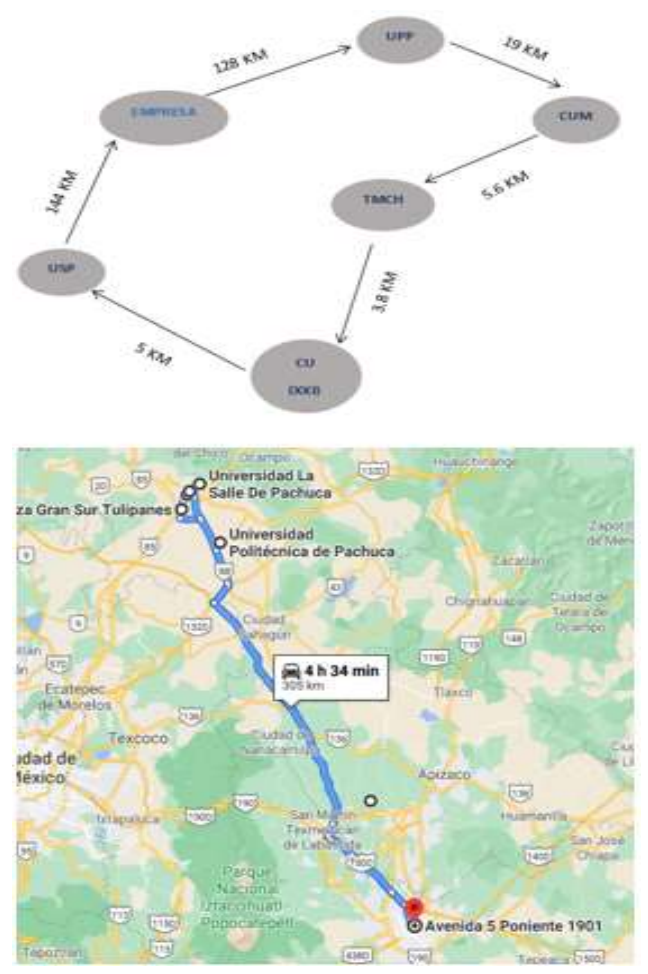
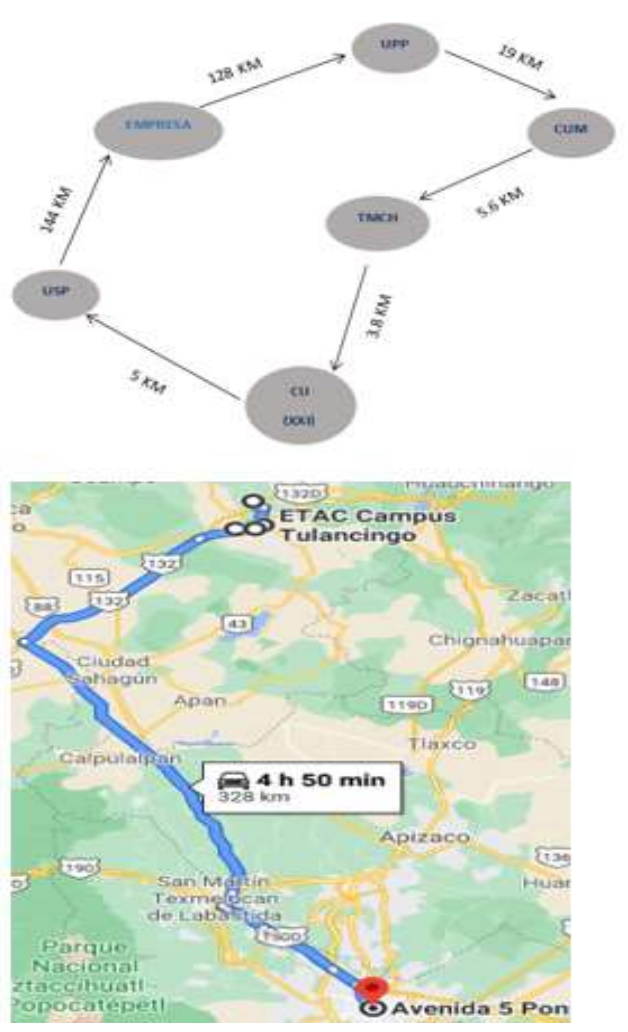

Fuente. Elaboración Propia con la aplicación del vecino más cercano.

Para la evaluación del resultado, la Tabla 6 presenta los datos generados con los recorridos antes y después de la aplicación de la técnica del vecino más cercano con apoyo de Google Maps.

Tabla 6 Comparación de los recorridos antes de la aplicación de la técnica del vecino más cercano.

\begin{tabular}{lcc}
\hline Variables de control & $\begin{array}{c}\text { Reporte } \\
\text { de Agente } \\
\text { Viajero }\end{array}$ & $\begin{array}{c}\text { Reporte Google } \\
\text { Maps y vecino } \\
\text { más cercano }\end{array}$ \\
\hline \hline Km recorrido total & 1,014 & 733.6 \\
Tiempo total empleado (minutos) & 1,440 & 684 \\
Costo de combustible & $\$ 1,560.00$ & $\$ 958.7$ \\
Viáticos para agente & $\$ 900.00$ & $\$ 450.00$ \\
\hline \hline Costo total & $\$ \mathbf{2 , 4 6 0 . 0 0}$ & $\mathbf{\$ 1 , 4 0 8 . 7}$
\end{tabular}

Fuente: Elaboración Propia. 


\section{Grafica 1 Comparación de las variables de control.}

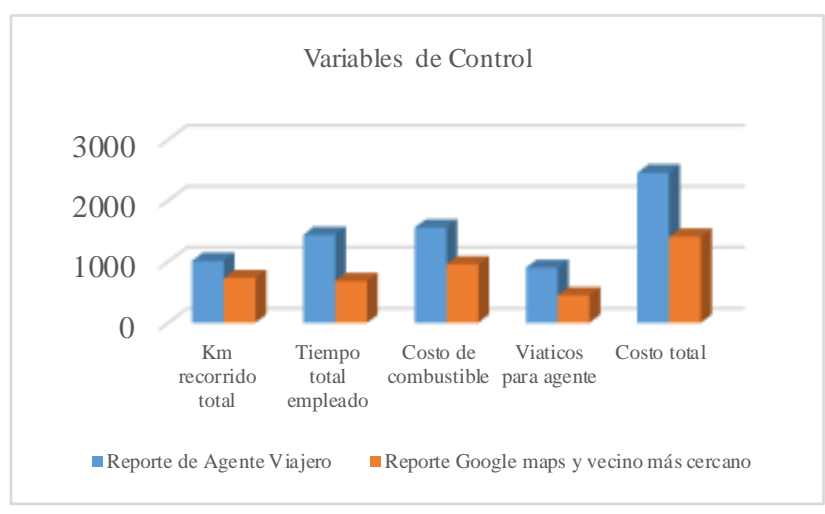

Fuente: Elaboración Propia.

La Gráfica 1 presenta las variables de control generando un ahorro del $27.7 \%$ en el $\mathrm{km}$., recorrido, para el tiempo empleado se obtuvo un ahorro del $52.5 \%$ ya que anteriormente la empresa argumentó que el agente realizó las 10 visitas en tres días con jornada laboral de 8 hrs., e incluso en ocasiones ya no regresó a la empresa ya que argumentaba falta de tiempo, por otra parte, con la aplicación de la técnica del vecino más cercano y la validación del km., y su seguimiento en google maps la primera ruta de recorridos de Pachuca se generó en un tiempo de $4 \mathrm{hrs} .34 \mathrm{~min}$. Y la de Tulancingo en un tiempo de $4 \mathrm{hrs} .50 \mathrm{~min}$., concediendo una hora de comida en cada recorrido, por lo que las visitas se generaron solo en dos días y el agente viajero regresó a la empresa a generarsu reporte informativo de trabajo. En cuanto a los viáticos en las primeras visitas antes de la aplicación del método, se le autorizó al agente un monto de viáticos por comidas de $\$ 300.00$ por día por lo que generó un gasto de $\$ 900.00$ en los tres día de visita, dado que en la aplicación del método la distancia y el tiempo se redujeron, al agente se le autorizó viáticos por $\$ 150.00$ por recorrido los cuales fueron realizados en 2 días por lo que acumuló un gasto de $\$ 300.00$ generando así un ahorro del $50 \%$, de esta forma en el costo de combustible se obtuvo un ahorro del $61.4 \%$ estableciendo un ahorro global en los costos de operación del $42.7 \%$.

\section{Conclusiones}

La aplicación de la técnica del vecino más cercano en combinación con el uso de las tecnologías de información Google maps para la solución del problema del agente viajero, para este caso permitió reducir los costos globales de operación en cuanto a los viáticos del agente, así como los costos del consumo de combustible, a razón de diseñar la ruta más corta para la visita a clientes minimizando la distancia y reduciendo el tiempo de recorrido, ofreciendo ventajas competitivas, garantizando su crecimiento y una competitividad en el mercado.

\section{Bibliografía}

Arias, J. A. (2017). Aplicación del problema de agente viajero a los principales hoteles de la ciudad de barranquilla.. ciudad de barranquilla: Research gate.

Arriagada, M. R. (2015). Comparación de métricas de distancia en el a lgoritmo K-Vecinos más cercanos para el problema de reconocimiento automático de dígitos manuscritos. En M. A. Rodríguez, Informe Del Proyecto Para Optar Al Título Profesionalingeniero De Ejecución En Informática (pág. 7). valparaiso: PONTIFICIA UNIVERSIDAD CATÓLICA DE VALPARAÍSO.

Battin, P. (2016). Location based. Reminder android application using google maps Api International conference on automatic control And dynamic optimization techniques (icacdot).

Chillagano, P. (2017). En P. Chillagano, Google maps como herramienta de sensibilización vial: un estudio en la escuela de conducción < <eccosytur >>. (págs. 11-15).

Cobos, D. (2014). Búsqueda y exploración estocástica. Revista de matemática: teoría y aplicaciones., 127-144.

Contreras, A. (2020). Impacto social-economico del transporte de carga en Mexico. En a.Contreras, Ingenio y conciencia boletin cientifico de la escuela superior ciudad Sahagun (págs. 32-38). Sahagun.

Cota,I. (25 de 04 de 2020). La economía de México se descompone a pasos agigantados. El pais. Recupera do el 15 de 03 de 2021, de https://elpais.com/economia/2020-04-25/la-economia-demexico-se-descompone-a-pasos-agigantados.html

Fuentes, A. (2016). Solución al Problema de Secuenciación de Trabajos mediante el Problema del Agente Viajero. ScienceDirect, 430 437.

Inegi. (2020). Encuesta Nacional De Ocupacion Y Empleo. Mexico: Nueva Edicion. Cuarto Trimestre.

López, C. (2013). "algoritmo para la exploración de todos los valores posibles en el problema del agente viajero (tsp)". scientia et technica 14

Mendoza.(2017). Travelling salesman problema (tsp) diseño de algoritmos heurísticos y metahuristicos eficientes para resolver el problema del agente viajero. Universidad nacional autónoma de Nicaragua.: Nicaragua, Managua.

Otamendi, R. (2014). Aplicación web de geo posicionamiento basada en la herramienta de google maps para la consulta de rutas de transporte publico $\mathrm{rn} \mathrm{rl}$ municipio de hatillo. municipio de hatillo.

Páez, B. K. (2016). Propuesta de diseño y desarrollo de una aplicación vinculada a google maps para la visualización de rutas vehiculares, dirigidos al sector de empresas de transporte de la ciudad de Guayaquil. ciudad de Guayaquil: Universidad de Guayaquil facultad de ciencias a dministrativas.

Ravea, J. I. (2012). Espacio literario relevante sobre el problema del vendedor viajero (TSP): contenido, clasificación, métodos y campos de inspiración. En J. I. Ravea, Espacio literario relevante sobre el problema del vendedor viajero (TSP) (págs. 5-6). bogota: PRODUCAO/ Universidad Nacional.

Reyes, A. J. (2017). Algoritmo del agente viajero para minimizar el recorrido de visitas programadas en una empresa de seguros. Revistas.uss.edu.pe, 5-6. 
Publicación semestral, Ingenio y Conciencia Boletín Científico de la Escuela Superior Ciudad Sahagún, Vol. 8, No. 16 (2021) 9-16

Rodriguez. (2016). Estrategias Para Transportar Bienes. La Importancia Del Transporte De Carga, 18-25.

Transporte, I. M. (2020). La dispersion del covid-19 a traves de os corredores de transporte carretero en Mexico. Mexico: Notas publicacion bimestralde divulgacion externa.

Universal. (08 de 04 de 2020). Quiénes y en dónde han perdido su empleo en México por el Covid-19. el universal. Recuperado el 15 de 03 de 2021 , de

https://www.eluniversal.com.mx/nacion/coronavirus-en-

mexico-quienes-y-en-donde-han-perdido-su-empleo

Villegas, F. J. (2017). Aplicacion Al Metodo Metz A La Solucion Del Problema Del Agente ViajerO. SCIENTIA ET TECHNICA, 341-344. 